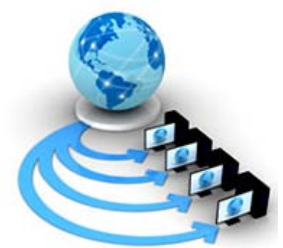

International Journal of Advanced Research in Computer Science

RESEARCH PAPER

\title{
A MULTI CONSTRAINED QOS ROUTING ALGORITHM FOR VEHICULAR ADHOC NETWORKS
}

\author{
Bharati S Pochal \\ Assistant Professor Dept of studies in computer applications \\ VTU center for PG studies, Kalaburagi \\ Karnataka, India
}

\author{
Shraddha K Sindhankeri \\ PG Student, Dept of studies in computer applications \\ VTU center for PG studies, Kalaburagi \\ Karnataka, India
}

\begin{abstract}
Secure Quality of Service (QoS) directing calculations are a crucial piece of remote systems that plan to give administrations QoS and security ensures. In vehicular specially appointed systems (VANETs), vehicles perform directing capacities, and in the meantime go about as end-frameworks subsequently steering control messages are transmitted unprotected over remote channels.
\end{abstract}

Keywords: Vanet's; Multi-Constrained Qos(MCQ); Secure Routing; Evolving Graph; ACO

\section{INTRODUCTION}

With the advancement of remote correspondence system, VANET has gotten extensive consideration on data sharing and information conveyance administrations.

VANET network is an uncommon type of Mobile Adhoc Network that gives both Roadside-to-Vehicle Transportations and vehicle-to-Vehicle Infrastructures administrations. So as to gather current activity condition and convey movement control data to vehicles, Brainy Transportations System needs to adequately utilize VANET and movement frameworks that comprising of all the available resources from the already installed hardware. Street Side Units are utilized for gathering movement measurable information, briefly buffering information, distinguishing vehicle current areas and shortening the correspondence delay. They are generally conveyed at altered areas (e.g., street crossing points Some VANET structures have been given in the procedure on the anticipated courses and calendars of transports all together to enhance the broadcast execution. In any case, none of them exploit the current movement frameworks.

\section{RELATED WORK}

In [1], Because of late improvements in remote correspondence systems, Vehicular_Adhoc_Networks advances have gotten a great deal of consideration in the fields of data sharing and administration revelation. In any case, because of the always moving portability of vehicle flat grid, data with the guide of open transportation frameworks. Transport courses can be utilized to make a spine structure on which information can be presented and circled on maintain a strategic distance from the show storm issue. The proposed engineering can adequately disperse and find the required information through the movement base and versatile vehicles. Test results show that the proposed plan outflanks different plans as far as parcel conveyance proportion and end-to-end delay. In addition, the problem of this plan is not exactly different plans with an expanding of number of administration solicitations. In
[2], this presents 3 vehicle direction based information sending plans, custom-made for vehicular impromptu systems. These days' GPS-based route frameworks are prevalently utilized for giving effective driving ways to drivers. With the driving ways called vehicle directions, we can make information sending conspires more proficient, considering the small scale perused portability of individual vehicles in street systems and the full scale checked versatility of vehicular movement insights. This paper indicates why the vehicle direction is a key fixing in the configuration of the vehicle-to-base, infrastructure to vehicle, and vehicle-to-vehicle information sending plans over multihop. Through the scientific plan, the key configuration strategies are appeared for three sending plans taking into account vehicle direction, contrasted and a cutting edge information sending plan in view of just vehicular movement insights. In [3], Multi-jump information conveyance through vehicular specially appointed systems is entangled by the way that vehicular systems are profoundly portable and every now and again detached. To address this issue, we receive convey and forward, where a moving vehicle conveys the parcel until another vehicle moves into its region and advances the bundle. Unique in relation to existing convey and forward arrangements, we make utilization of the predicable vehicle portability, which is constrained by the movement example and street design. In light of the current activity design, a vehicle can locate the following street to forward the parcel to decrease the postponement. We propose a few vehiclehelped information conveyance (VADD) conventions to forward the bundle to the best street with the most reduced information conveyance delay. Trial results are utilized to assess the proposed arrangements. Results demonstrate that the proposed VADD conventions beat existing arrangements as far as parcel conveyance proportion, information bundle postponement and convention overhead. Among the proposed VADD conventions, the H-VADD convention has much better execution. In [4], In sensor systems, it is essential to outline and utilize vitality effective correspondence conventions, since hubs are battery-fueled and accordingly their lifetimes are constrained. We propose 
an information spread convention for intermittent information redesigns in remote sensor systems, called SAFE (sinks getting to information from situations), which endeavors to spare vitality through information conveyance way sharing among various sinks that have regular interests. In [5], Arrange wide TV in Mobile Ad Hoc Networks gives vital control and course foundation usefulness for various unicast and multicast conventions. Considering its wide use as a building hinder for other system layer conventions, the MANET people group needs to institutionalize a solitary procedure that effectively conveys a bundle from one hub to all other system hubs. Notwithstanding a significant number of proposed television conspires, no extensive near investigation has been beforehand done. This paper gives such examination by grouping existing television plans into classes and recreating a subset of every classification, hence supplying a dense yet thorough next to each other correlation. The recreations are intended to pinpoint, in every class, particular disappointments to network conditions that are significant to MANETs, e.g., data transfer capacity blockage and element topologies. Also, convention augmentations utilizing versatile reactions to network conditions are proposed, actualized and investigated for one TV plot that performs well in the near study. In [6], A Trajectory-Based Data Forwarding (TBD) plan, customized for the information sending for street side reports in light-activity vehicular impromptu systems. Best in class plans have shown the adequacy of their information sending methodologies by abusing known vehicular movement insights (e.g., densities and rates). These outcomes are empowering, be that as it may, further changes can be made by exploiting the developing notoriety of GPSbased route frameworks. This paper shows the main endeavor to successfully use vehicles' direction data in a security safeguarding way. In our outline, such direction data is joined with the vehicular activity measurements for a superior execution.

\section{SYSTEM DESIGN}

\section{A. Proposed System}

We propose another BUS_VANET design which completely coordinates activity foundations with transports and vehicles. Here we take into account focal points of R_S_Us and T_C_C that as of now been given by ITS to enhance the VANET's execution. In view of the given architecture BUS_VANET, we utilize the enrolment innovation to enhance the transmission execution and give another strategy for selecting enlistment hub to decrease the quantity of changes from regular vehicles to high-level hubs. We likewise proposed another plan for recognizing the destination area all the more proficiently.

\section{Advantages of Proposed system}

- Increase packet delivery ratio.

- Decrease security overhead.

- Decrease security vulnerabilities.

- $\quad$ Provides the Quality of Service.

\section{B. objectives of the study}

- It ought to distinguish the destination area all the more productively.

- Higher conveyance rate and shorter conveyance delay.

- Lower danger of security break.

\section{Methodology Used}

- Information conveyance will be done by the partnership of transports, RSU and Traffic Control Centre (TCC). The versatile transports and altered area RSUs are powerfully shaping an associated topology.

- The anticipated courses and calendars of transports keeping in mind the end goal to enhance the transmission execution. Be that as it may, none of them exploit the current movement frameworks.

- The administration scope by transports can't be ensured and there is no proficient destination area distinguishing proof technique created in customary VANET. Along these lines, we are going for planning another VANET engineering that completely coordinates transports and activity frameworks for giving better information conveyance administrations.

- $\quad$ RSUs can be utilized to make up for the deficiency of transports once in a while to guarantee the administration scope while TCC can be useful for finding the destination vehicle rapidly.

\section{System Architecture}

Frameworks configuration is the strategy for characterizing the outline, modules, components, interfaces, and learning for a framework to fulfill indicated needs. Frameworks configuration can be seen in light of the fact that the use of frameworks hypothesis to improvement.

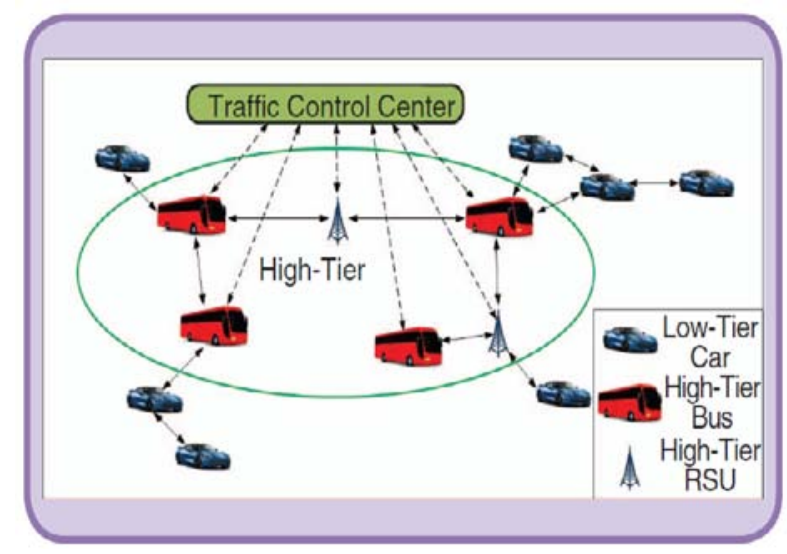

Figure 1: Basic Architecture of the proposed work

Figure 1, Demonstrates that the improvement of vehicular specially appointed systems (VANETs). VANETs are a specific type of remote system complete by cars communicating with R_S_U. The remote correspondences given by VANET's can possibly encourage new administrations that could spare a great many exists and enhance the pouring knowledge.

\section{IMPLEMENTATION}

\section{A. Modules Description}

1) System Configuration :

In the principal module we build up the Network Configuration for our proposed model. A few critical 
upgrades are presented in our new two-level design since we completely coordinate with activity frameworks. Firstly, 3 presumptions are made in our system

a) All cars, transports and R_S_Us are outfitted with D_S_RC gadgets for speaking with among themselves and G_P_S based route framework with a computerized guide. Current data about movement insights is likewise accessible to them.

b) Busses and RSUs are also furnished with Wimax correspondence ability. Along these lines, they are really framed a spine of VANET.

c) The course and timetable of each transport and the area of each RSU are imparted to every single other vehicle.

\section{2) Choice of Registration Node:}

All cars have to enlist with a close-by high-level hub for getting information conveyance administration. Step by step instructions to Figureure out which transport or RSU ought to be chosen for enrolment is a vital issue if a vehicle got a few guides from various high-level hubs.

At the point when a vehicle got a dynamic guide from a transport or RSU_Unit, this transport or R_S_U will be viewed as a hopeful enrolment high-level hub and be given into a competitor set. In the event that a vehicle lost association with its at present enrolled transport or R_S_U, it needs to change its enlistment to additional high-level hub.

Since changing starting with one transport or RSU_unit then onto the next will bring about way re-calculation and reconstructing, we go for diminishing the quantity of such switches.

\section{3) Vehicle place Finding:}

By coordinating different system with transports and vehicles, we plan another plan for recognizing the terminus vehicle rapidly. we provide more insights about this control system distinguishing proof plan counting how to locate the right area of a destination and how to diminish the working of T_C_C. As we specified, every transport or R_S_U keeps an enlistment vehicles are right now enrolled. These enrollmenttables_Vehicle will be accounted for to the TCC intermittently and TCC keeps up an area table to store these gathered data.

\section{4) Performance Evaluation:}

This is very important module of the system, where one can find out the results and can be compared with the current system. There are two ways to compare the results, graphical ways and analytical ways. Both can be used, but in system we have used xgraph way to compute the graph and show the output.

\section{PERFORMANCE ANALYISIS}

\section{A. Tools and Technology}

This chapter describes general aspect and technologies used in this project. Before developing any model we should collect background information of the model. The general aspect includes overview of wireless network, Application of wireless network, Characteristics of wireless network. Coming to technology part, it consists of network simulator (NS-2), NAM and utility graph. A Wireless Sensor Network (WSN) comprises of generally circulated free sensors to check a portion of the natural or physical conditions, similar to weight, temperature, sounds, and so forth furthermore move their data through a system to fundamental area. The most modern recent now are twodirectional, also able to monitor sensor working condition. Making of WSN was because of defense applications, but currently it is used for so many other applications like science and engineering. WSN has grown very much, and one can see this technology is being used in all departments. From government offices to the private company everyone is taking the benefit of this great technology which is shaping the world towards comfort and quality services. WSN has grown form limited uses to many health care business environments. Present day such type of NW are used for company and manufacturing units.

- Zone monitoring

- Natural/Earth monitoring

- Air contamination monitoring

- Timberland fire detection

- Water quality monitoring

- Common calamity prevention

- Machine wellbeing monitoring

\section{B. Results and Discussion}

RTA broadcast encrypted master key to all RSU:

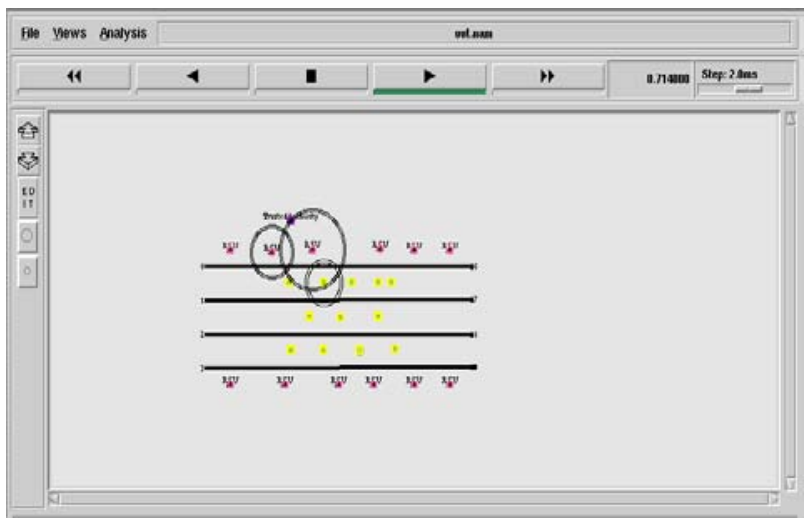

Figure 2. Shows the service of Vanet.

The service of Vanet is divided into three components i.e. a Vehicle, RSU and RTA. In this the RTA can broadcast the encrypted master key to all RSU for Security of Data transmissions from source to destination.

\section{Vehicle to RSU Authentication:}

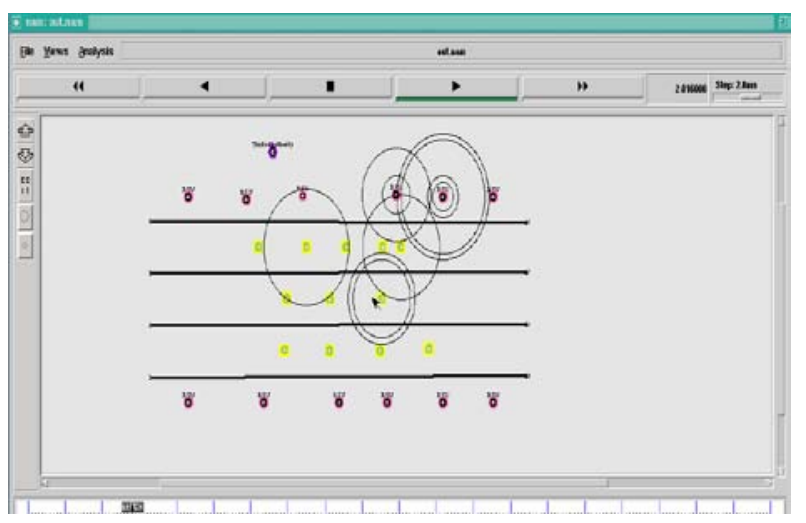

Figure 3. Shows the Vehicle Communication. 
Figure 3 shows that how the Vehicle (nodes) can communicate with all RSU for authentication purpose and each vehicle can request the master key request to the RSU for Secure vehicular communication among each vehicles.

\section{Vehicle to Vehicle (V2V) Authentication:}

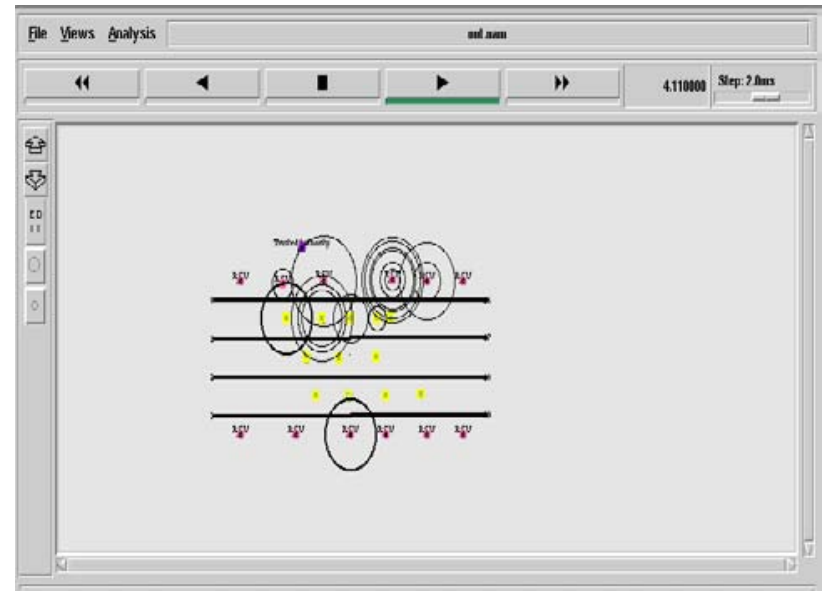

Figure 4. Vehicle to Vehicle Authentication (V2V).

Figure 4 Shows that the Vehicle to Vehicle Authentication (V2V).i.e. each vehicle can be authenticated among themselves for searching feasible route from source to destination vehicles.

\section{The Source Vehicle 5 Search the feasible route:}

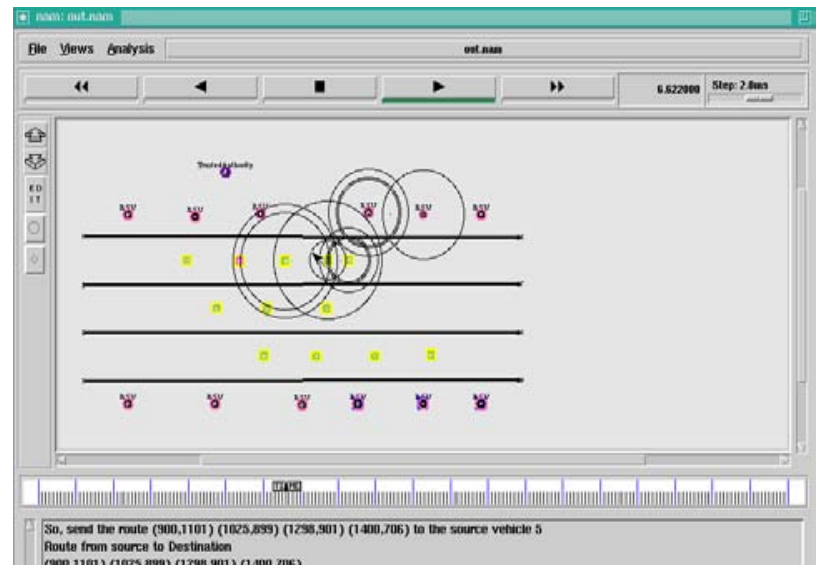

Figure: 5 Feasible Route Search.

Figure 5 Shows that, The Source Vehicle (5) can search the feasible route for secure and reliable way of data transmissions from source to destination vehicle (15).

Route from Source (5) to Destination (15):

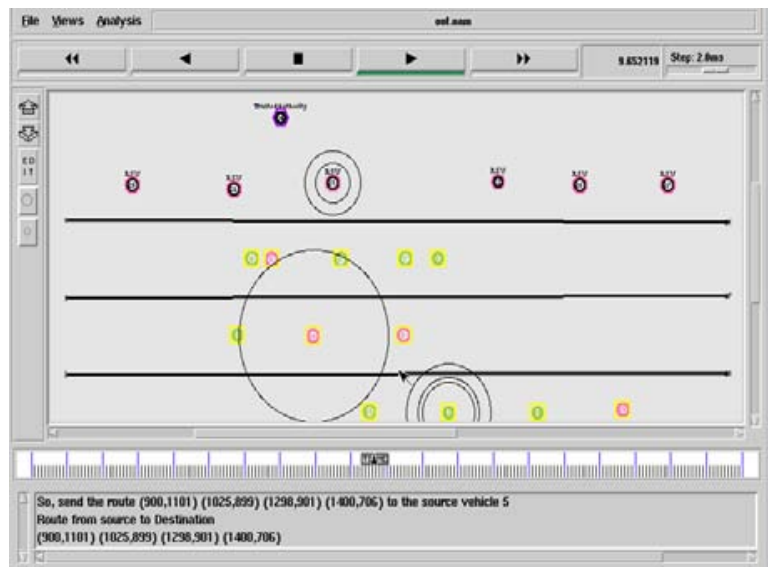

Figure: 6 shows that the final path for the data transmission.

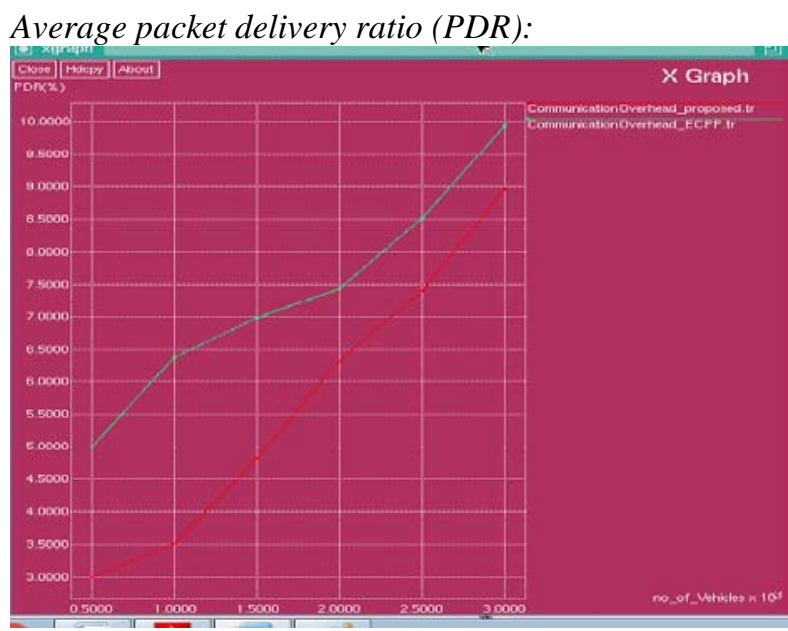

Figure 7. Average Packet Delivery Ratio.

Figure 7 demonstrates that, The Average packet delivery ratio which speaks to the normal proportion of the no. of effectively got information packets at the goal hub to the no. of information bundles sent.

Average time for route discovery:

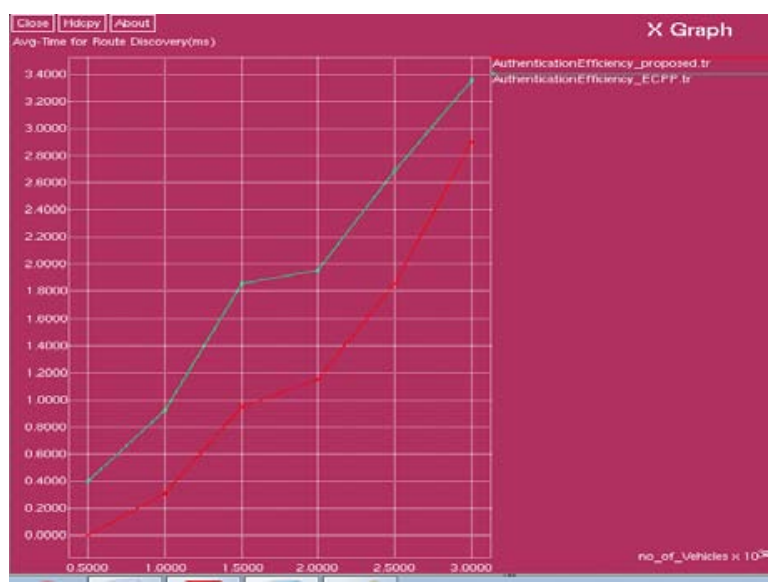

Figure 8, Route Discovery.

Figure 8 demonstrates that, The Average time for course disclosure which speaks to the time expected to play out the course revelation prepare from source to goal. 


\section{QoS Routing}

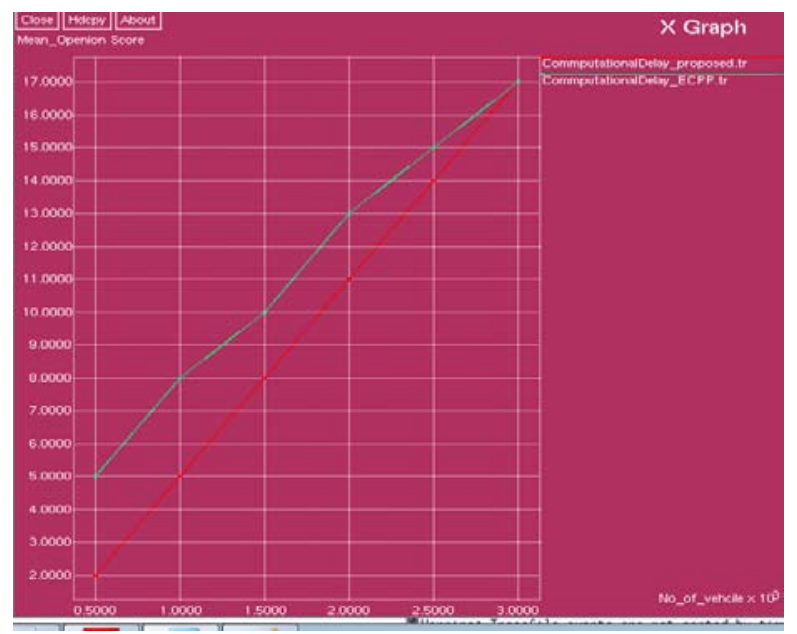

Figure 9. Shows that, how the QoS Routing from source to destination.

\section{Mean Opinion Score (MOS)}

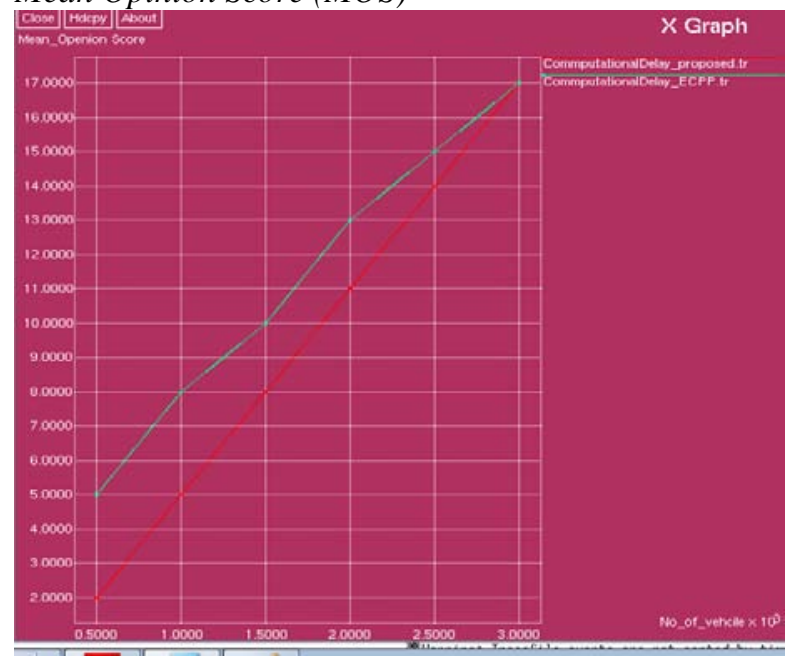

Figure 10. Demonstrates that, The Mean Opinion Score which speaks to the QoS routing from source to destination.

\section{CONCLUSION}

We have shown another two-level BUS-VANET that completely incorporated with activity bases for enhancing the execution of VANET. We exploit RSUs_BUS VANET and TCC_BUS VANET that officially required and developed by IT'S and researches the amount of advantages we can acquire from this reasonable environment by coordinating infrastructure of the road side and vehicles which are moving on the road. Using the available resources one can develop the application in less cost with transports, the scope of the high-level hubs can be guaranteed and the likelihood of parcels conveying is decreased.

\section{REFERENCES}

[1]. R. Yu, Y. Zhang, S. Gjessing, W. Xia, and K. Yang, “Toward cloud based vehicular networks with efficient resource management,’'IEEE Netw.Mag.,Vol. 27,no.5,pp. 4854,sep./oct.2013.

[2]. K. Yang, S. Ou,H. Chen, and J.He,”Amultihop peercommunication protocol with fairness guarantee for IEEE 802.16-based vehicular networks,”IEEE Trans. Veh. Technol., Vol. 56,no. 6,pp. 3358-3370,Nov. 2007.

[3]. Z. Wang and J. Crow croft, "Quality of service routing for supporting multimedia applications," IEEE J. select. Areas Comm.,vol.14,no.7,pp.1228-1234,Sep. 1996

[4]. D.S. Reeves and H.F.Salama,"A distributed algorithm for delay constrained unicast routing," IEEE/ACM Trans. Netw., vol.8,no.2,pp.239-250,Apr. 2000.

[5]. Y. Bejerano,Y. Breitbart, A. Orda,R. Rastogi,and A. Sprintson,"Algorithms for computing QoS paths with restoration," IEEE/ACM Trans. Netw.,vol. 13,no.3,pp.648661,Jun.2005.

[6] B. Zhang, J. Hao, and H. T. Mouftah, "Bidirectional multiconstrained routing algorithms," IEEE Trans. Comput., vol. 63, no. 9,pp.2174-2186,sep.2014.

[7] P. Van Mieghem, H. D. Neve, and F. A. Kuipers, "Hop-byhop quality of service routing,” Comput. Netw., vol. 37, no. 3/4,pp. 407-423, Nov. 2001.

[8] G. Liu and K. G. Ramakrishnan, "A_prune: An algorithm for findingk shortest paths subject to multiple constraints," in Proc. IEEE20th Annu. Joint Conf. IEEE Comput. Commun. Soc., Anchorage,AK, USA, 2001, vol. 2, pp. 743-749.

[9] T. Korkmaz and M. Krunz, "Multi-constrained optimal path selection," in Proc. IEEE 20th Ann. Joint Conf. IEEE Comput. Commun.Soc., Anchorage, AK, USA, 2001, vol. 2, pp. 834-843.

[10] P. Van Mieghem and F. A. Kuipers, "Concepts of exact QoS routing algorithms,” IEEE/ACM Trans. Netw., vol. 12, no. 5, pp. 851-864, Oct. 2004. 